\title{
Wie wirksam ist die Instillation von Alteplase?
}

\author{
Für Patienten, die komplizierte parapneumonische \\ Pleuraergüsse (PPE) und Pleuraempyeme (PE) entwickeln, ist \\ die intrapleurale Fibrinolyse ein therapeutischer Fortschritt. \\ Der Plasminogenaktivator Alteplase besitzt Eigenschaften \\ eines idealen Fibrinolytikums. G. Thommi et al. haben die \\ Wirksamkeit und Sicherheit von Alteplase nun überprüft. \\ Respir Med 2012; 106: 716-723
}

An der palcebokontrollierten, randomisierten, doppelblinden Studie konnten Patienten mit PPE und PE teilnehmen, die sich Dekortikationen oder videoassistierten Thorakoskopien unterziehen sollten. Alteplase $(25 \mathrm{mg}$ in $100 \mathrm{ml}$ Kochsalzlösung) wurde täglich über 3 Tage intrapleural instilliert. Alle Patienten erhielten über die Studiendauer Breitbandantibiotika. Patienten des ersten Studienarms, die auf die Therapie nicht ansprachen, konnten sich entweder chirurgisch weiter behan- deln lassen oder in einem zweiten Studienarm die medikamentöse Behandlung (Alteplase oder Placebo) fortführen. Nebenwirkungen wurden über mindestens 24 Stunden kontrolliert. Der primäre Endpunkt war eine $40 \%$ ige Reduktion chirurgischer Eingriffe von Placebo zu Alteplase.

Insgesamt beendeten 68 Patienten die Studie. Sie waren im Mittel 64 Jahre alt (21-90 Jahre) und 37 von ihnen waren Männer. Bei 17 Patienten hatten sich Em- pyeme und bei 51 komplizierte PPEs entwickelt. Bei 58 von 61 mit Alteplase behandelten Patienten kam es zur Heilung (95\%). Das war bei 4 von 33 Patienten $(12 \%)$ unter Placebo der Fall $(p<0,001)$. Von den Patienten mit Empyem, die Alteplase erhielten $(n=16)$, erreichten alle Heilung, unter Placebo $(n=5)$ dagegen keiner $(p<0,001)$. Bei komplizierter PPE war die Verumtherapie in $93 \%(n=42$ von 45$)$ und die Placebogabe in $13 \%$ ( $n=4$ von 28$)$ der Fälle wirksam. Von allen Patienten wählte keiner die chirurgische Therapie. In Bezug auf Nebenwirkungen ergaben sich zwischen Verum und Placebo keine deutlichen Unterschiede.

\section{Fazit}

Die Studienergebnisse belegen im Placebovergleich eine hohe Wirksamkeit von Alteplase bei komplizierten parapneumonischen Pleuraergüssen und Pleuraempyemen. Die intrapleurale Instillation des Plasminogenaktivator erwies sich als sicher, die Nebenwirkungen blieben auf Placeboniveau.

Matthias Manych, Berlin

ren die Emissionen an VOCs und ultrafeinen Partikeln beim Konsum von E-Zigaretten geringer als bei der klassischen Zigarette“, sagt Schripp. Auch konnten der Forscher und sein Team bei E-Zigaretten keine Freisetzung von Formaldehyd nachweisen. Bei herkömmlichen Zigaretten hingegen wurde der Richtwert von 0,1 ppm (parts per million) für die Innenraumluft überschritten. Das Nebelfluid Propylenglykol entwich aus E-Zigarette sowie Tabakzigarette in die Raumluft, da es ebenfalls ein häufig verwendeter Zusatzstoff im Tabak ist. „Die E-Zigarette ist eine schwächere Quelle für Raumluftverunreinigungen als die Tabakzigarette, allerdings ist auch sie nicht emissionsfrei. Man kann daher davon ausgehen, dass Umstehende dem freigesetzten Dampf passiv ausgesetzt sind“, resümiert Schripp die Ergebnisse der Messungen. Mit der Studie wollen die Wissenschaftler orientierende Messwerte für weitere Untersuchungen vorlegen. „Eine toxikologische Einschätzung liefern wir damit jedoch nicht", betont Schripp.

Nach einer Mitteilung der FraunhoferGesellschaft, München 